\title{
Online Feedback System for Public Speakers
}

\author{
Anh-Tuan Nguyen, Wei Chen, Matthias Rauterberg \\ Designed Intelligence Group, Department of Industrial Design \\ Eindhoven University of Technology \\ Eindhoven, Netherlands \\ \{a.nguyen, w.chen, g.w.m.rauterberg\}@tue.nl
}

\begin{abstract}
The development of Affective Computing has witnessed tremendous number of studies about facial and vocal expression, while bodily expression only comprises the minority. However, with the emerging of social signal processing, people have paid more attention to the significant role of body language in social communication and emotional expression. One situation when body language shows its importance is public speaking. This paper presents an online feedback system for public speakers, in which emotion recognised from body language of speakers is regarded as the primary component for analysis. The system captures input through a Microsoft Kinect, thus offer users with a convenience way to interact. In order to recognise bodily expression, a posture and gesture representation method based on Laban Movement Analysis was adopted. The system could be used as a support tool for people who learn presentation skills, as well as for public speakers who need immediate feedbacks. This paper will present the architecture of the system, along with details about the employed methods and a collected database of presentations.
\end{abstract}

Keywords-emotion detection; social signal processing; technology enhanced learning.

\section{INTRODUCTION}

Research in human emotion was established since the 19th century via the classical works of Darwin [1] and James [2]. Later on, it emerged to the field of computing, remarked by the book of Picard [3], which established a new field, namely Affective Computing. The book has set up a well-accepted standard for building emotional intelligent systems, in which emotion detection systems have taken the largest proportion. Practically, the role of Affective Computing has been more and more recognized and found its position in the other fields like human-computer interaction [4], e-learning [5] and social signal processing [6].

Basically, emotion detection systems capture signals from people and then raise appropriate information about their emotional states. These signals could be taken via face, voice or body's expression. However, the attention spent for these kinds of signals has not been equal. The article in 2009 of Gelder [7] stated that $95 \%$ of the studies in human emotion had been conducted using facial expression stimuli while the rest $5 \%$ had been accounted by research using information from voice, music and body expression, in which research on wholebody expression comprising the smallest number. Recently, thanks to the advances in motion capture equipment, especially low-price gaming devices like Microsoft Kinect or Nintendo Wii, emotion detection from body language has been acquiring increasingly important role.

This work was supported in part by the Erasmus Mundus Joint Doctorate in Interactive and Cognitive Environments (ICE), which is funded by the EACEA Agency of the European Commission under EMJD ICE FPA n 20100012
One of the most noticeable situations when both body language and emotion have shown their significance is public speaking. Most people might agree that, public speaking is not only about what, but also how the information is delivered, in term of bodily or vocal expression. These expressions, as discussed in [8], are strongly affected by emotion. Actually, regarding emotion of public speakers, there are two ways of understanding: one is their emotional expression that could be perceived by the audience, and the other one is their internal emotion, which might be expressed or not. Obviously, by looking from the point of the audience, emotional expression, rather than the internal emotion, is the signal that could be used to analyse the performance of a speaker. By other words, good speakers are the persons who have the ability to show the desired (mostly positive) emotional expression, regardless their true internal feeling. Indeed, this task is not easy, leads to the great number of books and training courses about presentation skills.

Understanding the importance of presentation skills training from universities, companies, organisations and individuals, this research was conducted with purposes are simplifying and decreasing the cost spent for the training tasks through an automatic system. Therefore, we implemented an automatic feedback system for public speakers, which can be used by people who learn presentation skills, as well as professional speakers, who need immediate feedbacks during real presentations. The system has been built with three main requirements. First, user interaction must be friendly and convenience enough to avoid distraction to public speakers. Thus, a Microsoft Kinect was employed to capture data. The device offers us with rich description of human motion and allows users to interact naturally. Second, the feedbacks given to users should be informative but not distractive. This is a non-trivial issue and would be done in further processes of the project. Third, emotion expression through body language is regarded as the primary concern when building the system, since it is the most important cue to assess a speaker.

The paper is organised as follows. In the next section, we will review recent research related to the topic, including the assessment of public speaking, how emotion affect public speakers, and existing methods for emotion detection from body language. Detail about the system is presented in the following section, in which we show the architecture and the method for body posture and gesture representation. Moreover, the database of presentations from a presentation skills class has been collected and presented in this section. The last sections are for the future work and conclusion. 


\section{PREVIOUS RESEARCH}

In the literature, there are countless number of books telling us how to become a successful presenter, and also how to express the appropriate emotional expression during a presentation. This section will summarize the characteristics of a good presentation, and then take a look at existing systems for automatic assessment of public speaking. Since the system regards body language as the primary signal for assessment, recent advance in emotion detection from body language [9] will also be reviewed.

\section{A. Assessment of Public Speaking}

In general, apart from aspects concerning the content of presentations, performance of a speaker could be assessed through either verbal or non-verbal signals. Verbal signals consist of physical characteristics of speech such as tempo, pitch and articulation, while non-verbal signals mostly include information from body gesture, posture, facial expression and eye contact. In comparison between them, non-verbal signals have been confirmed that having stronger influence than verbal signals [10].

Emotion in public speaking has been studied long ago by psychologists [11], [12]. Most research has focused on the anxiety state of speakers, how this state is expressed in term of both visual and vocal signal, as well as how easily could it be recognized by the audience. Unfortunately, Winton [13] revealed that, audience become more active in recognition of negative emotional states of presenters, rather than the positive ones. Another research [14] studied the changed in EEG signal and heart rate of speakers and concluded that, both signals changed collectively account for $48 \%$ of the variance in the increase of negative affect. Nevertheless, emotion in public speaking is not just about avoiding negative emotional expression, but also about how to use emotion as a tool to empower your delivery, as stated in [8]: '... So, to reach people, you need to express proper emotion...' Indeed, successfully express emotion through your voice and body language increases the chance of success.

In the literature, there are several approaches toward automatic assessment of speakers during presentation, in order to raise appropriate feedback and serve as support tool for public speakers. To the best of our knowledge, there are two existing systems sharing this purpose [15], [16]. In [16], the authors directly analysed vocal signal of speaker via low-level physical characteristics. On the other hand, the research in [15] paid more attention on emotion expression of speakers. In fact, this study is mostly emotion detection from voice, rather than a speaker support system. The drawback of both systems is that, they only got cues from speakers' voice, although voice is less importance than body language in a presentation [9].

\section{B. Emotion Detection from Body Language}

Body language only takes the minor proportion in emotion detection [7], partly due to the lack of a general framework for body motion representation [17]. In the literature, emotion is detected through either posture or gesture. Research on postural emotion detection is still limited as static configuration of human body does not provide enough information to present the complexity of emotion. The most remarkable work in this direction was done by Kleinsmith [18]. The authors built a complicated representation of human body based on distances and angles of joints. On average, $70 \%$ of affective postures were correctly classified from a set of 111 samples. Another work of Schindler [19] detected emotion directly from images of postures. He applied image processing techniques, turned the problem into pattern classification and then got the recognition rate at $80 \%$ for 6 basic emotions. These systems for postural emotion detection although could achieve high recognition rate, still limited while there were many constrains required and the experiments were conducted in well-control environment.

On the other side, emotion detection from body gesture has captured greater attention. Most systems take input data from motion capture equipment, such as the VICON system [17] or gaming devices like Nintendo Wii [20] because these devices could provide great details about body motion. The most noticeable work in gestural emotion detection has been done by Camurri and the Infomus Lab [21]. This work, however, only used data captured from regular 2D cameras. The author proposed a four-stage framework, which adapted the Effort component of Laban Movement Analysis [22] as a body motion representation method. Experiments showed that recognition rate of the framework was as good as spectators [23]. Although the author provided a completed framework for bodily emotion detection, the work is still limited when can only work well when detect emotion from very limited objects (dancers and music performers). Besides that, there are number of studies takes Laban's theory for gestural emotion detection, such as [24] or [25]. However, none of them has succeeded in providing a unified method for interpreting low-level representation of body motion to Laban' components.

In general, emotion detection from body language is still limited, since there has been no general framework for this task. However, putting in the context of public speaking, bodily emotion detection is highly demanded. Next section will present an online feedback system, in which we proposed our own methods for posture and gesture representation.

\section{ONLINE FEEDBACK SYSTEM FOR PUBLIC SPEAKERS}

This section explains the system for automatic assessment of public speaking. Differ from previous systems that take speech signal into account [15], [16], this system focuses on body language of speakers. Other aspects need to be considered when assess a public speaker are the context and content of the presentation. In most case, speakers need to adjust the so-called degree of exaggeration [8] in order to fit with these aspects. For example, a public speaking for president election requires much greater exaggeration than for a class lecture. President candidates must use stronger gestures, louder, slower voice, wider eyes contact and so on, if they want to reach the audience. Similarly, presenting the same content for a small group of 10 people should not use as much exaggeration as for a large group with 500 people. The feedback system is studied in the context of small conference room when the number of audience is from 15 to 20 people. Content of the presentation would be about scientific topics that are new to most audience. 
In that case, main purpose of the speaker would be transferring new information and let the audience being interested.

Furthermore, since most bodily expression of speakers is motored by emotion, as discussed in the previous section, emotion expression is regarded as an important indication for performance of public speakers. Details about the system are explained as follows.

\section{A. System Description}

Figure 1 shows the diagram of the system, which includes the capture device and two processing modules: (1) Emotional Signals Detection and (2) Performance Assessment. Details about each component are explained as follows.

A Microsoft Kinect was implemented in our project as capture equipment. The device offers us with both colour video and motion information (through its built-in skeletisation module). Vision-based device was chosen instead of other methods like wearable sensors with purpose to bring friendly interaction mechanism for users. In fact, choosing visual capture equipment offered some possibilities: (1) 2D regular cameras, which there has been already a well-developed framework, namely the Eyesweb framework, introduced by Camurri [21]; and (2) 3D capture systems, which employed in [25]. Since the feedback system would like to capture explicit representation of body, 2D image processing would not provide enough input data, as revealed in [26]. On the other hand, 3D vision systems require high computation complexity, as well as inconvenience calibration processes, thus not suitable for the system.

The Emotional Signal Detection module is the heart of the feedback system. It takes data from Kinect and recognises emotional signals from body language of speakers. We propose to pay more attention to recognition of negative emotional states such as fear or nervous, since the audience are more sensitive to them [13]. The module includes two sub-modules for performing separated tasks: (1) Posture Analysis and (2) Gesture Analysis, respectively. Finally, detected emotion is processed in the Performance Assessment module, which produces final decision about the performance of the speaker and gives appropriate feedbacks. Next section will explain how these modules have been implemented, as well as how our database has been collected for analysis.

\section{B. Collection of Database}

Development of the system requires a database of recorded presentations. These samples must be recorded and annotated with assessments about the performance of speakers, serving as ground truth. In the project, sample data was collected from the course 'The Art of Presenting Science', which is given for all of employees in our university. During the course, learners were asked to give short presentations (approximately one minute), and then got feedbacks from the trainers about their performance, as well as what should they do in order to be improved. The feedbacks are in high degree of details. For example, the posture of learners was good/bad, the gesture should be more/less, how hand movement should change, etc. We have been recoding both these presentations and feedbacks as ground truth. There have been 32 presentations recorded.

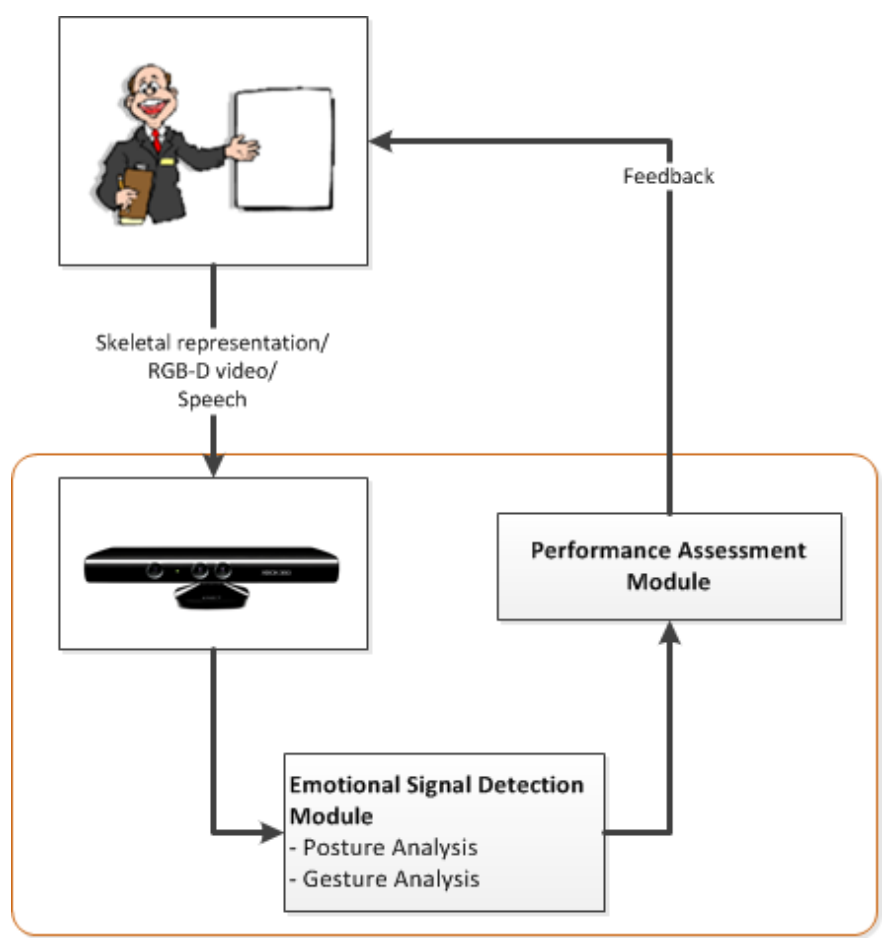

Figure 2. Architecture of the System

\section{Posture Analysis}

In the system, posture analysis is required since specific static configuration of public speaker could reveal some information about their emotion, thus leads to judgments about their performance. Using Microsoft Kinect as input equipment, along with its built-in skeletisation module, provides great details about 3D positions of body joints. Therefore, we proposed a posture representation method, built upon angles between body parts. Reason for taking angles into account, instead of distances between joints (as [27]) is that, we have looked forward scale-invariant characteristic as users can be various in their bodies' size. 3D angles were projected in three planes, thus gave three feature components for each angle. These angles are listed in Table 1. Upper part of the body was focused on since it can provide more information about emotion. Finally, a body posture was represented as a 15component vector.

In presentation skills, there are some specific good and bad postures. This representation method is supposed to be able to classify these postures, therefore give indication about

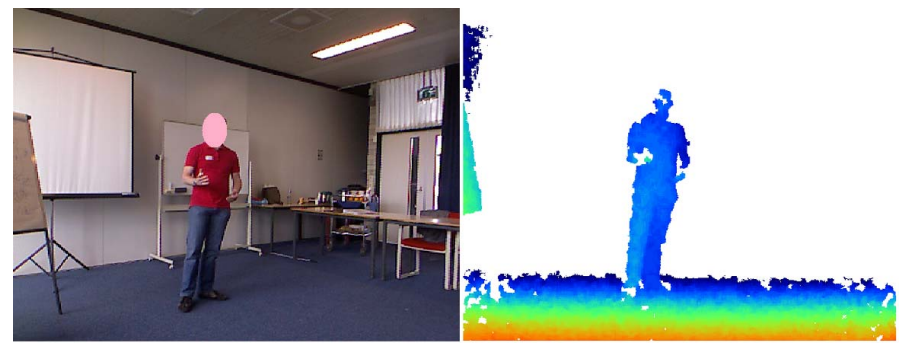

Figure 1. One sample from the database. (Left) color image; and (Right) depth image 
TABle 1. Features of Posture RePresentation

\begin{tabular}{|l|c|}
\hline \multicolumn{1}{|c|}{ Angles between } & Number offeatures \\
\hline Upper and lower arms & 6 \\
\hline Upper arms and shoulders & 6 \\
\hline $\begin{array}{l}\text { Shoulder and ground plane } \\
\text { (the balance of human body) }\end{array}$ & 1 \\
\hline $\begin{array}{l}\text { Face and shoulder, projected to ground floor } \\
\text { (durection of human head, horizontally) }\end{array}$ & 1 \\
\hline $\begin{array}{l}\text { Face and ground floor } \\
\text { (how height the person is looking at) }\end{array}$ & 1 \\
\hline
\end{tabular}

TABLE 2. CORRESPONDENCE BETWEEN LMA COMPONENTS AND PHYSICAL ENTITIES

\begin{tabular}{|l|c|}
\hline LMA parameters & Physical entities \\
\hline Space & Motion Histogram \\
\hline Effort.Time & Acceleration, Velocity \\
\hline Effort.Space & Curvature, Angular velocity \\
\hline Effort.Weight & Acceleration \\
\hline Effort.Flow & Curvature, Angular velocity \\
\hline
\end{tabular}

speakers' performance. A set of both good/bad postures was collected through reviewing the database. These postures were built upon the moments where the trainers said that the postures were good/bad. The classification has been done simply through the nearest-neighbour algorithm.

\section{Gesture Analysis}

More attention has been paid on gestural characteristic of body language, since it offers richer representation via dynamic configuration. Related to this task, gesture and body language recognition have been well developed, especially for Social Signal Processing [6]. However, they would not be suitable because emotion expression of public speaker requires higher degree of abstraction, rather than some specified gestures. Therefore, we looked at Laban Movement Analysis (LMA) [22] as a prominent method for body motion representation. The book of Bartenieff [22] provided a unified view about LMA, which includes four components: Body, Effort, Shape and Space. In the literature, the Effort component is mostly used because it links directly to the internal attitude for movement of people, thus offers reliable indications about emotion. The Effort component itself contains four subcomponents: Time, Space, Weight and Flow.

We have implemented a method to interpret skeletal movement of body into LMA's components. In fact, most relevant works in the literature have aimed to find the correspondence between physical entities of movements with a particular set of LMA's components, e.g. [25], [28]. The work of Rett [25] has offered significant indications. In this work, Laban.Space was linked to angular displacement and Laban.Effort was linked to curvature, velocity and acceleration of two hands. Our system inherited the interpretation of Laban.Effort. For Laban.Space, motion histogram was employed, with purpose is to give better representation about the movement of body in 3D space. The correspondence
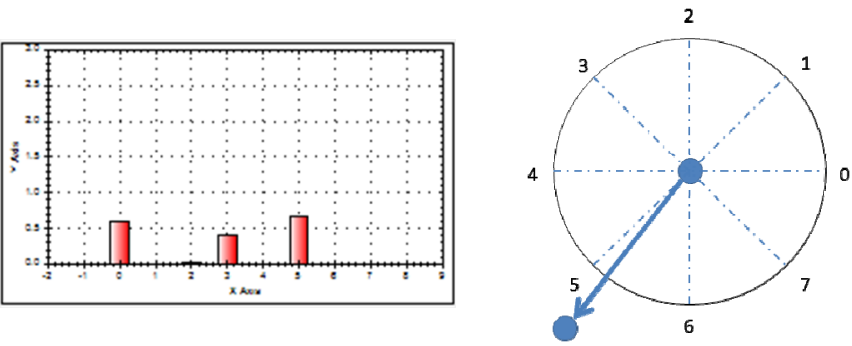

Figure 3. One histogram and its computation for one joint. In this example, bin number 5 was chosen since it was closest to the displaement vector

between LMA's components and physical entities in our system is summarized in Table 2.

Motion histogram is the method widely used in gesture recognition [29]. It has been considered as a simple but effective algorithm in terms of body motion representation. Basically, motion histograms are built by firstly detects direction of movement within a specific time frame, and then the amount of movement is accumulated into the appropriate histogram bins. In our project, 8-bin motion histograms are created for every joint, after their displacements are projected on three planes. Thus, we can remain a feature vector with 480 components during the analysis [20 joints provided by Kinect $\mathrm{x}$ 8 histogram bins $\mathrm{x} 3$ planes].

On the other hand, estimation of Laban.Effort was more straightforward since the parameters like acceleration or velocity of two hands could easily be calculated via the $3 \mathrm{D}$ positions. For curvature, we used the degree of changes in $3 \mathrm{D}$ angular displacement as the estimation.

\section{E. Assessment of Public Speaking and Feedback}

When a representation for posture and gesture has been generated, a method for classification is necessary. The literature of machine learning offers a great number of algorithms for classification [30]. Choosing an algorithm for this purpose must be relevant to the form of feedbacks that the system would like to give users. Our system proposed a simple form of feedback, with purpose to give enough information but do not disturb the presentation. We have designed feedbacks in simple visual form that is displayed on computer screen. The feedbacks show the assessment about performance of users in terms of their posture and gesture, through a scale of five degrees [Bad - Not bad - Neutral - Good - Excellent].

\section{CONCLUSION AND FUTURE WORK}

In this paper, we present our online support system for public speakers. The system could be used by people who learn presentation skills, as well as professional presenters who need immediate feedbacks. Using Microsoft Kinect as input device, the system offers users with friendly interaction method, and feedbacks are given in such a way that informative but simple enough to not disturb presenters. Emotional expression via body language has been regarded as the key component for the assessment. For emotional body language recognition, the system employed a method for posture representation that built upon angles between body segments. Gesture was represented through two components of the Laban Movement Analysis: 
Space and Effort, in which Space was estimated by motion histogram and Effort was estimated from spatial displacement of body joints. Combining posture and gesture, a representation of body language was generated, which proposed to be informative enough to assess performance of public speakers.

Current state of the system has mostly focused on the representation of body language, since there has been no standard framework for this task. We have not spent much effort for classification as there has been great number of existing methods available in the field of machine learning. In the future, the system needs to be tested in real situation, where a particular classification algorithm would be employed. Furthermore, the representation would be able to enrich by adding information from other aspects such as facial expression, eye contact and voice. These aspects have been proved to be informative in assessment of public speaking, thus are proposed to be able to integrate with cues from postures and gestures to form a united feedback system.

\section{REFERENCE}

[1] C. Darwin, The expression of the emotions in man and animals, 2nd ed. London: , 1980.

[2] W. James, "What is an Emotion?," Mind, vol. 9, no. 34, pp. 188-205, 1884 .

[3] R. W. Picard, Affective Computing, 1st ed. The MIT Press, 2000.

[4] E. Hudlicka, "To feel or not to feel: The role of affect in humancomputer interaction," International Journal of Human-Computer Studies, vol. 59, no. 1-2, pp. 1-32, Jul. 2003.

[5] L. Shen, M. Wang, and R. Shen, "Affective e-Learning : Using 'Emotional' Data to Improve Learning in Pervasive Learning Environment Related Work and the Pervasive e-Learning Platform," Learning, vol. 12, no. 2, pp. 176-189, 2009.

[6] A. Vinciarelli, M. Pantic, and H. Bourlard, "Social signal processing: Survey of an emerging domain," Image and Vision Computing, vol. 27, no. 12, pp. 1743-1759, Nov. 2009.

[7] B. de Gelder, "Why bodies? Twelve reasons for including bodily expressions in affective neuroscience.," Philosophical transactions of the Royal Society of London. Series B, Biological sciences, vol. 364, no. 1535, pp. 3475-84, Dec. 2009.

[8] C. Reimold and P. Reimold, The Short Road to Great Presentations: How to Reach Any Audience Through Focused Preparation, Inspired Delivery, and Smart Use of Technology, 1st ed. Wiley-IEEE Press, 2003.

[9] J. D'Arcy, Technically Speaking: A Guide for Communicating Complex Information. Battelle Press, 1998.

[10] A. W. Siegman and S. Feldstein, Nonverbal bahavior and Communication, 2nd ed. Psychology Press, 1987, pp. 37-64.

[11] P. Feldman, S. Cohen, and N. Hamrick, "Psychological stress, appraisal, emotion and cardiovascular response in a public speaking task," Psychology \& Health, 2004.

[12] T. Freeman and C. Sawyer, "Behavioral inhibition and the attribution of public speaking state anxiety," Communication Education, no. March 2012, pp. 37-41, 1997.

[13] E. Winton, D. Clark, and R. Edelmann, "Social anxiety, fear of negative evaluation and the detection of negative emotion in others," Behaviour Research and Therapy, vol. 33, no. 2, pp. 193-196, 1995.

[14] R. J. Davidson, J. R. Marshall, a J. Tomarken, and J. B. Henriques, "While a phobic waits: regional brain electrical and autonomic activity in social phobics during anticipation of public speaking.," Biological psychiatry, vol. 47, no. 2, pp. 85-95, Jan. 2000.

[15] T. Pfister and P. Robinson, "Real-Time Recognition of Affective States from Nonverbal Features of Speech and Its Application for Public Speaking Skill Analysis," Affective Computing, IEEE Transactions, pp. 1-14, 2011.
[16] D. Silverstein and Tong Zhang, "System and method of providing evaluation feedback to a speaker while giving a real-time oral presentation, US Patent 7,050,978,' U.S. Patent US Patent 7,050,9782003.

[17] D. Bernhardt, "Emotion inference from human body motion," University of Cambridge, Computer Laboratory\$\$, 2010.

[18] A. Kleinsmith, T. Fushimi, and N. Bianchi-Berthouze, "An incremental and interactive affective posture recognition system," in Context, 2005, pp. 1-13.

[19] K. Schindler, L. Van Gool, and B. de Gelder, "Recognizing emotions expressed by body pose: a biologically inspired neural model.," Neural networks : the official journal of the International Neural Network Society, vol. 21, no. 9, pp. 1238-1246, Nov. 2008.

[20] N. Savva, "Automatic recognition of affective body movement in a video game scenario," in International Conference on Intelligent Technologies for interactive entertainment INTETAIN11, 2011, vol. 44.

[21] G. Castellano, S. D. Villalba, and A. Camurri, "Recognising Human Emotions from Body Movement and Gesture Dynamics," Network, pp. 71-82, 2007.

[22] I. Bartenieff, Body movement: Coping with the environment. CRC Press, 1980.

[23] A. Camurri, "Recognizing emotion from dance movement: comparison of spectator recognition and automated techniques," International Journal of Human-Computer, vol. 59, pp. 213-225, 2003.

[24] T. Lourens, R. Van Berkel, and E. Barakova, "Communicating emotions and mental states to robots in a real time parallel framework using Laban movement analysis," Robotics and Autonomous Systems, vol. 58, no. 12, pp. 1256-1265, 2010.

[25] J. Rett, "Robot-human interface using Laban Movement Analysis inside a Bayesian framework," University of Coimbra, 2008.

[26] G. Castellano, M. Mortillaro, A. Camurri, and G. Volpe, "Automated analysis of body movement in emotionally expressive piano performances," Music Perception, vol. 26, no. 2, pp. 103-119, 2008.

[27] A. Kleinsmith and N. Bianchi-Berthouze, "Recognizing affective dimensions from body posture," Affective Computing and Intelligent, pp. 48-58, 2007.

[28] T. Lourens and E. Barakova, "Retrieving emotion from motion analysis: In a real time parallel framework for robots," Neural Information Processing, pp. 430-438, 2009.

[29] J. Davis, "Recognizing movement using motion histograms," Technial Report 487, MIT Media Lab, vol. i, no. 487, 1999.

[30] L. Kaelbling and M. Littman, "Reinforcement learning: A survey," Journal of Artificial Intelligence Research, vol. 4, pp. 237-285, 1996. 
(C) 2012 IEEE. Personal use of this material is permitted. However, permission to reprint/republish this material for advertising or promotional purposes or for creating new collective works for resale or redistribution to servers or lists, or to reuse any copyrighted component of this work in other works must be obtained from the IEEE.

\section{IEEE Symposium on E-Learning, E-Management and E-Services}

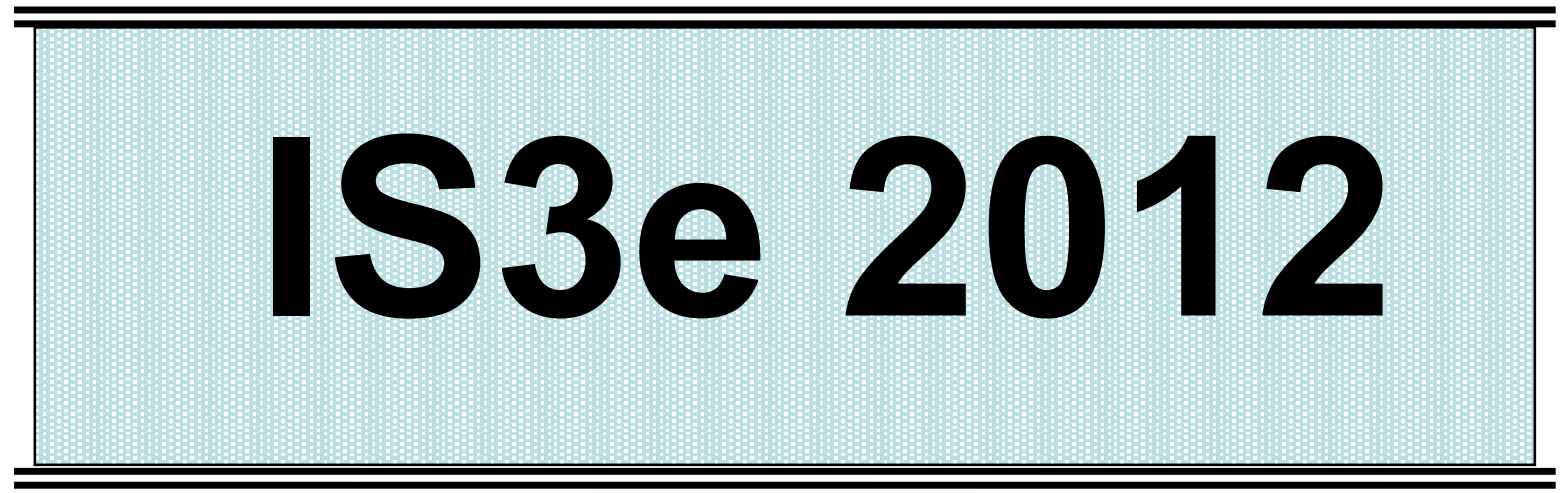

IEEE Catalog Number : CFP12IS3-CDR

ISBN : 978-1-4673-2388-8

CD-ROM Support: IS3e2012 Secretariat Phone: (+603) 55211198 Email: mycs@computer.org

Sponsors

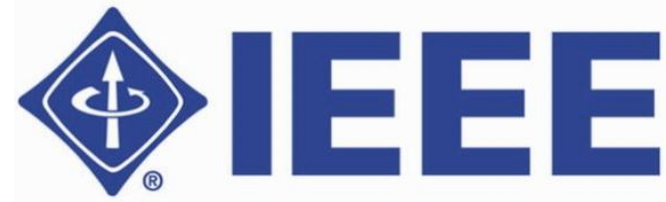

IEEE Computer society 
Copyright and Reprint Permission: Abstracting is permitted with credit to the source. Libraries are permitted to photocopy beyond the limit of U.S. copyright law for private use of patrons those articles in this volume that carry a code at the bottom of the first page, provided the per-copy fee indicated in the code is paid through Copyright Clearance Center, 222 Rosewood Drive, Danvers, MA 01923. For other copying, reprint or republication permission, write to IEEE Copyrights Manager, IEEE Operations Center, 445 Hoes Lane, Piscataway, NJ 08854. All rights reserved. Copyright @2012 by IEEE. 


\section{WELCOME ADDRESS}

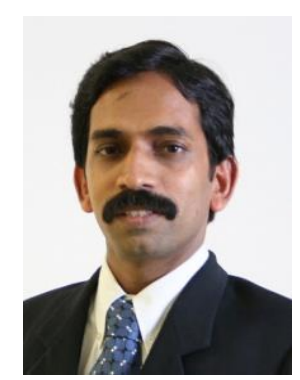

\section{General Chair, ICOS 2012 and IS3e 2012}

Distinguished Presenters and Participants,

It gives me great pleasure to welcome all guests, speakers and participants to Kuala Lumpur to attend the IEEE Conference on Open Systems (ICOS) 2012 and IEEE Symposium on e-Learning, eManagement and e-Services (IS3e) 2012 organized by IEEE Computer Chapter, Malaysia Section.

The ICOS 2012 aims to provide an excellent opportunity to share and exchange technologies and applications in the area of Open Systems for professionals, engineers, academics and industrial people worldwide. The IS3e 2012 symposium will be an ideal platform for researchers, engineers and application developers to share views and experiences in e-Learning, e-Management, e-Services research, products and services.

I would like to thank our distinguished speakers, Prof. Dr. Tony Wasserman (Carnegie-Mellon University, USA), Prof. Dr. Mohamed Amin Embi (UKM) and Mrs Tan King Ing (MAMPU) for their commitments and dedications. I would also like to take this opportunity to thank the workshop presenters. A special thanks to International Advisory Panel members for their invaluable ideas and contributions to these conferences.

I would like to give a special gratitude and appreciation to ICOS 2012 and IS3e 2012 committee members and IEEE Computer Society members who have contributed and work very hard to make these conferences a successful one.

It is our hope that ICOS 2012 and IS3e 2012 can help to shape a community that will lead to greater innovations in the area of Open Systems, e-Applications and e-Services.

I am sure the ICOS 2012 and IS3e 2012 conferences will be of great benefit to you and hope you will enjoy your stay in Malaysia.

Mohamed Rawidean Mohd Kassim

General Chair, ICOS 2012 and IS3e 2012 